\title{
A Novel Hybrid Secure Routing for Flying Ad-hoc Networks
}

\author{
Dr. Jennifer S. Raj, \\ Department of ECE, \\ Gnanamani College of Technology, \\ Namakkal, India. \\ Email: jennifer.raj@gmail.com.
}

\begin{abstract}
The recent technology development increases the opportunity to create valuable network services to user. Flying ad-hoc networks (FANET) in one among them which evolved recently with enhanced value added services with common features similar to its predecessor ad-hoc networks like vehicular ad hoc networks (VANET) and mobile ad hoc networks (MANET). Due to its distinctive features FANETs are widely preferred in recent telecommunication services which requires high quality of services, efficiency, environment adaptability and scalability. In order to achieve high efficiency multiple aerial vehicles are used in general architectures. The Communication in such vehicles are progressed directly between the nodes or through relay nodes. Routing is an important process to establish a connection link between the nodes in the architecture. This research work proposed a routing strategy suitable for dynamic and static environments as a hybrid optimization model which reduces the issues in link establishment. Nature inspired bee colony optimization is used along with conventional routing algorithms such as optimized link state routing protocol and Dynamic Source Routing Protocol to improve the link discovery. The proposed optimized routing outperforms well in reduced delay and communication overhead of the network.
\end{abstract}

Keywords: - Flying Ad hoc Networks, Routing, Active and Reactive topology.

\section{Introduction}

Mobile ad hoc networks (MANETs) are widely used in various applications and it is further expanded into Flying ad hoc networks (FANETs) as an improved version which uses flying machine for transmission and reception in wireless communication. The advantages of FANETs is its easy setup and wireless communication realization in anyplace. It has multi-hop, self-organizing, self-healing characteristics similar to its predecessor MANET. Using small group of flying vehicles this network could be deployed rapidly and the nodes are coordinated from the ground which gives high degree of freedom compared to any other networks. Unmanned aerial vehicle network is discussed earlier to bring this effective flying ad hoc networks. This flying networks helpful in complex situations where the conventional network could not be established due to natural calamities. The wide range of applications of FANETs will be disaster management, rescue operations, security and other location aware services. Earlier single aerial vehicle network based systems are evolved, but these systems have lagged performance due to its network scalability and limited capability operations. These makes the system inflexible and non-adoptable for wide range of applications. Later multi-layer aerial vehicle networks are evolved to reduce the issues in single aerial vehicle networks and this is termed as flying ad hoc networks. Due to tis infrastructure less network characteristics it doesn't require any central control system and its swarms themselves in air and arrange a wireless network. Without any centralized system the elements in the network communicates with each other and also to the base station which is an added advantage in flying ad hoc networks. FANETs are widely used in various applications like calamity administration, location aware services, sensor networks, Military services and other rescue operations. Figure 1 gives an illustration of flying ad hoc networks in detail. 
Vol.02/ No. 03

Pages: $155-164$

https://www.irojournals.com/tcsst/

DOI: https://doi.org/10.36548/jtcsst.2020.3.005

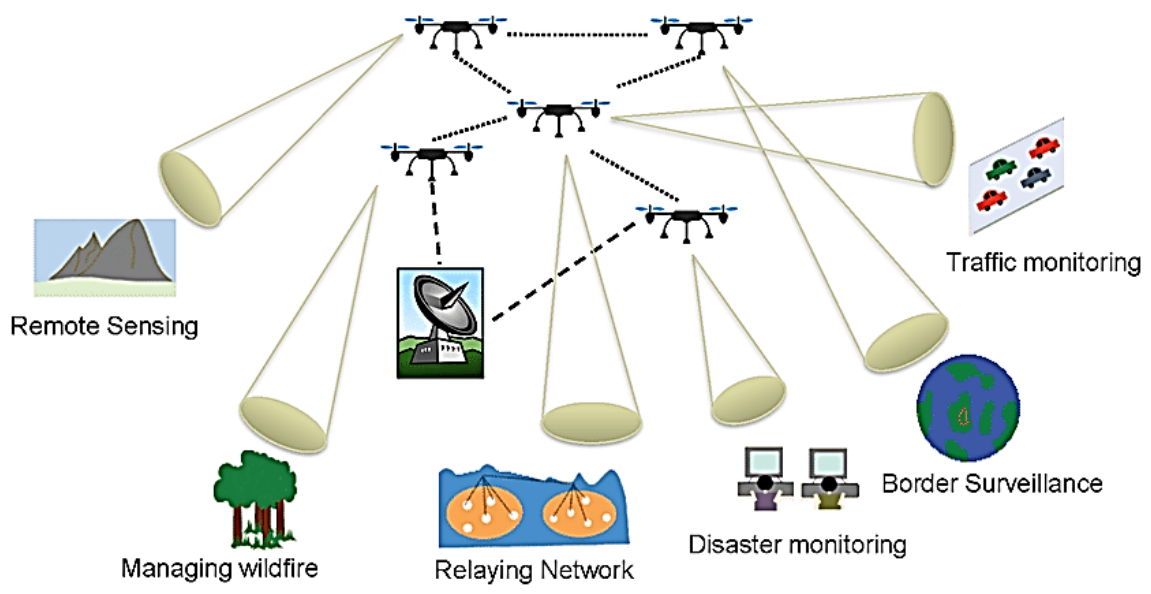

Fig. 1 Flying Ad hoc Network (FANET)

The characteristics of aerial vehicles as nodes in flying ad hoc networks are fast and it has high degree of opposition due to its restricted transmission link bandwidth and switching characteristic. This will lead into network complexity and error rate. In a dynamic situation, FANETs have strong mobility and easily lead into route breaks, also the dynamic update of routes leads into high overhead to the network which increases the risks in route convergence. Due to this large overhead, network may face issues like increased forward delay and packet loss which leads the routing protocol into inefficient. Realizing effective network routing for the high dynamic flying ad hoc network is a challenging task. Routing in FANET is similar to MANET routing but the routing protocol must adapt the changes in the network with respect to high speed nodes. Table 1 gives an important difference between Since routing is an important factor for any wired or wireless network, this research work concentrates on routing strategy in FANETs.

Table 1 Comparison of Ad Hoc Networks

\begin{tabular}{|c|c|c|c|c|}
\hline S.No. & Characteristics & VANET & MANET & FANET \\
\hline 1 & Node Density & Medium & Low & Low \\
\hline 2 & Node Mobility & Medium & Low & High \\
\hline 3 & Mobility & Steady & Arbitrary & Predetermined \\
\hline 4 & Network Topology Change & Average & Slow & High and Rapid \\
\hline 5 & Computational power & Average & Limited & High \\
\hline 6 & Network lifetime & Average & Limited & Limited \\
\hline
\end{tabular}

Various routing protocols are available for wireless ad hoc networks which are on demand, dynamic, cluster based routing, etc., Since FANET is a class of MANET and VANET, the existing routing protocols are used to test the performance. But, due to the aerial vehicle and its rapid changes most of the routing protocols are not adoptable for FANETs. The recent advancement and its improvements makes the routing process easier in FANET. The routing protocol in FANET is broadly classified into six categories. Figure 2 depicts the classification of routing protocols. 
Journal of trends in Computer Science and Smart technology (TCSST) (2020)

Vol.02/ No. 03

Pages: $155-164$

https://www.irojournals.com/tcsst/

DOI: https://doi.org/10.36548/jtcsst.2020.3.005

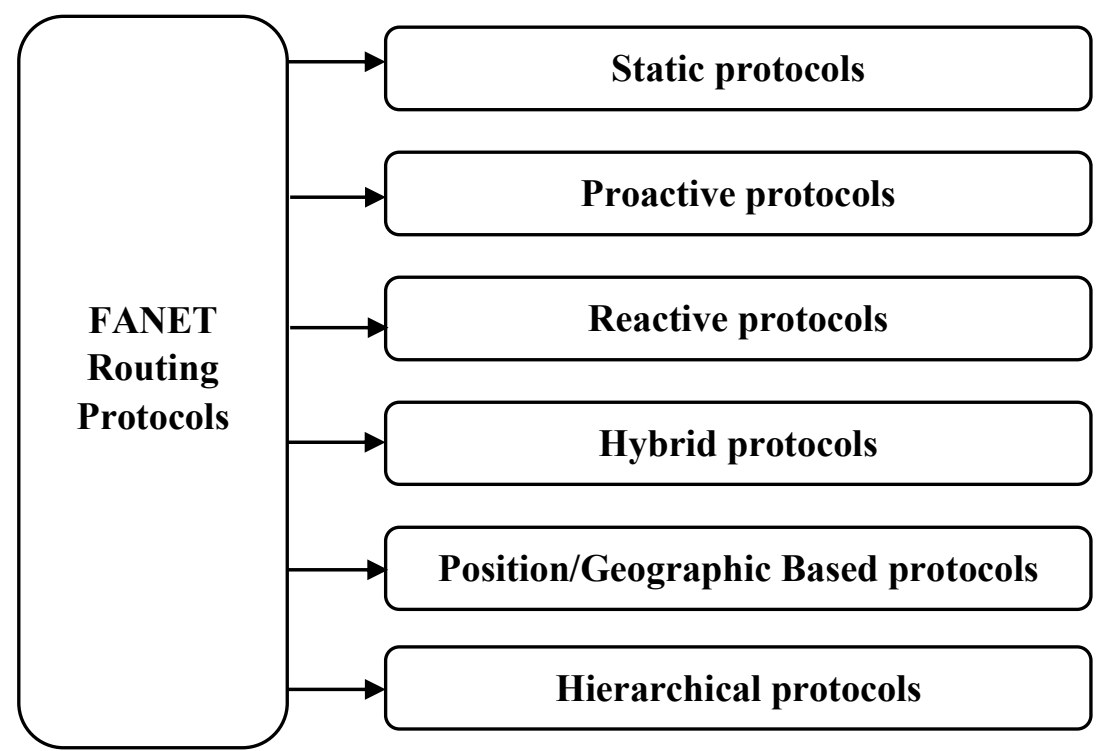

Fig. 2 Routing Protocols in FANET

In this static protocols uses fixed routing tables and the routing link is established based on the values in the table. In case of proactive the tables which has node details are frequently refreshed and the positions are updated. The reactive protocols are on demand protocols which establishes routes based on the requirement. It discovers the message path based on the demand between the nodes. If the protocol uses both reactive and proactive routing strategy, then it is hybrid protocol. Base on the position and the area coverage the path is established in position or geographic based protocols. The last hierarchical protocol uses hierarchy model for efficient routing process in flying ad hoc network. Using these six categories of routing protocols, FANETs identifies the path for node communication and provides efficient services.

\section{Related works}

The issues in existing routing protocols and its methodologies are considered as key points for analysing vast literatures related to FANET routing. Based on the categories the analysis is performed and issues are summarized in this section for motivation of the proposed routing model. İlker Bekmezci.et.al. [1] discussed about various routing protocols in FANET. In this static routing protocol, a predetermined routing table is designed and uploaded to the nodes before network initialization and once the network is initialized then the routing strategy could not be altered. The communication between the nodes and the base station is limited in static routing protocol and it could be changeable after completion of the assignment which is considered as limitation of static routing protocol. Chaker Abdelaziz.et.al. [2] discussed about data centric routing in FANET which has option to support single to multiple data transmission. Using on demand algorithms, the data requested nodes are selected for data transmission while the other nodes are not considered for transmission process. Similar static routing model as multilevel hierarchical model is presented in Vishal Sharma.et.al. [3] research model which uses hierarchical clusters to organize and operate the network. Cluster heads are used to represent the entire cluster and this cluster heads are responsible communication between the nodes and aerial vehicles. Communication is entirely depending in the cluster heads and if the cluster head loses its energy then the communication is disturbed until the next cluster head is selected. The is considered as a limitation in this hierarchical model.

Wajiya Zafar.et.al. [4] reported the issues in static routing protocol and suggested the proactive routing protocol which is suitable for dynamic environments. Similar to static protocol, proactive also uses tables but the table was frequently updated and it has the most recent values of paths so that he communication becomes easy. Proactive model greatly reduces the transmission delay, but it requires large number of communication between the nodes to update the table which requires large bandwidth. This large bandwidth requirement is considered as limitation of proactive routing protocol. Ashish Khanna. et.al.[5] suggested a directional optimized link state routing which uses multi point relay nodes. These multi point relay nodes could be used by more than a node which reduces the bandwidth requirement in conventional model and reduces the communication overhead to the base station. But the limitation of link stage routing is its number of multi point relay units which increases the transmission delay in the network. Rayner.et.al. [6] discussed the advantages of sequenced distance vector routing 
Journal of trends in Computer Science and Smart technology (TCSST) (2020)

Vol.02/ No. 03

Pages: $155-164$

https://www.irojournals.com/tcsst/

DOI: https://doi.org/10.36548/jtcsst.2020.3.005

in which routing table is saved in all the nodes and any changes in the nodes are updated in the table and circulated to the other nodes as updated position. This updating process eliminates the loops in routing process and identifies the recent and correct routes for communication. The limitation of sequence distance vector routing is its table updating process and periodic broadcast of table status leads into increased network overhead.

Reactive protocols are on demand routing protocols and it doesn't have any communication between the nodes. This reduces the route calculation process between the nodes which reduces the communication overhead to the network. Min Zhang.et.al. [7] discussed the importance of reactive routing protocol through dynamic source routing based on wireless networks. The source node in dynamic source routing considers all the neighbour nodes and sends a request for route information and it got multiple messages. In order to differentiate the node messages each message is accepted with its id and the essential route is established. It has a unique route repair mechanism to update the changes in the network is an added advantage in dynamic source routing. Similar to distance source routing, Inam Ullah Khan.et.al. [8] proposed an ad-hoc on demand distance vector routing which uses single data table for each node which reduces the multiple entries issues faced in distance source routing. It uses the source node to store the neighbour node information for data communication and performs routing through route discovery, route maintenance and packet transmission. Ad-hoc on demand distance vector is modified based on time slot is reported in Ali Khan.et.al. [9] research model which is similar to ad-hoc on demand vector model with dedicated time slots to transfer data packets from one node to other node. The limitation of time slot model is its increased bandwidth usage but the proposed model increases the packet delivery ration and reduces collisions.

The issues in proactive and reactive models are overcome by hybrid routing protocols which uses both reactive and proactive protocols in a single network. The entire network is divided into various zones and the inter zones are processed with reactive models and the intra zone communication is performed through proactive routing protocols. Imtiaz Mahmud.et.al. [10] reported the issues in conventional proactive and reactive routing protocols and proposed a zone routing protocol for efficient communication. In which based on the minimum distance and radius the zones are predefined and data communication is performed considering the node position. If the node position is inside the zone, it is considered for proactive routing process and if the node is outside the zone, then it is considered for reactive routing protocol. Armir Bujari.et.al. [11] discussed the advantages of temporarily ordered routing algorithm which preservers the routes and the router information of neighbour nodes. In most of the cases temporarily ordered routing models uses reactive routing strategy and very rarely used proactive routing. This routing algorithm uses directed acyclic graphs to preserve the information and communicates from source to destination using shortest path. This reduces the network overhead by assigning a height value in graph for the nodes which causes data flow from one to other node. The limitation of these model is its loop free structure which cannot provide guarantee for data flow from higher node to lower node.

Position based routing is reported in Jiaxin Chen.et.al. [12] research model which essentially requires the position of the node and calculates the location through positioning facilities like GPS. In this position based routing the sender has to decide and use the facility to select the destination node. Also, it uses forwarding methods for data packets forwarding process which increases the packet delivery ratio. Nishat.et.al. [13] discussed the importance of routing process in FANET through greedy perimeter stateless routing which is based on position based routing. Compared to other conventional routing protocols this perimeter based routing has various advantages such as high efficiency and mobility. The limitation of this routing is its reliability due to its perimeter based operation. Yixin He.et.al.[14] discussed the importance of hierarchical routing in FANETs which will be a choice for routing based on the hierarchical level. The initial route establishment is performed through proactive routing models and based on the requests in the node it is converted into reactive routing process which are performed in lower level of the network. Network complexity and node identification are the complex issue in hierarchical routing process. Under hierarchical routing, mobility prediction and clustering algorithms are evolved which improves the routing performance, but it is not up to considerable level so that it is not widely preferred to complex operations. From the above survey it is observed that routing is an essential process in FANET and most of the routing algorithms are concentrated over node mobility. High bandwidth and communication overhead are the limitations of few research models and none of the models are discussed the security issues in FANET. Based on these observations the proposed model is designed to achieve reliable routing for efficient communication with improved security in flying ad-hoc networks.

\section{Proposed Work}

The proposed model is designed as hybrid model which used optimized link state routing and dynamic source routing for link analysis and artificial neural network is used to classify the link status which is further optimized through artificial bee colony optimization for efficient link management. A short overview of the routing protocols, neural network models are discussed before proceed into the proposed design. The optimized link state 
Journal of trends in Computer Science and Smart technology (TCSST) (2020)

Vol.02/ No. 03

Pages: $155-164$

https://www.irojournals.com/tcsst/

DOI: https://doi.org/10.36548/jtcsst.2020.3.005

routing protocol is a familiar routing protocol in ad-hoc networks which is proactive model. It maintains the node details as tables which has details of entire nodes in the network. Due to this feature optimized link state routing protocol is widely used in routing strategies in the wireless networks. The main features of link state routing are its reduced size of control packets and multi-point relay nodes which are used to communicate with other nodes in the network. Using these multi point relay nodes in the network reduces the message scattering the network which greatly reduces the network traffic and bandwidth requirement. In case of dynamic source routing, it has effective route discovering ability from the network. Dynamic source routing is a reactive type routing model which obtains the node and route details from the header by combining multiple information. Using node network interface and route details from neighbour nodes, it produces more network topologies which improves the route maintenance property in wireless networks.

The route discovery in the routing algorithm starts if there is a requirement of data transmission from a node. Once the request is received, the route discovery process is initiated and it checks the destination which is present in the same zone or from the different zone. Once it is analysed and finds the destination details then the route link is initiated and a connection is established between the nodes using routing protocol. If the destination node is in same zone, then optimized link state routing is established and if the destination is in another zone then dynamic source routing is used for routing process. once the link is established, immediately a table is created in the source node which updates the route details and the route is maintained continuously to monitor the network topology. The routing table is continuously updated throughout the process and the network changes are continuously monitored throughout the process. If the network faces any route break, then an error packer will be send to the source node which defines the status of data transmission and destination node status and deleted the route information from the table and again this process is repeated to find next possible route to start the data transmission. In order to classify the link status and maintain the link a three-layer artificial neural network is introduced in the proposed model. The output of each neuron is given as

$$
y_{n}=\rho \sum_{i=1}^{n} h_{i j} x+v
$$

Where $\rho$ is the neuron activation function, $h$ is the weight, $x$ and $v$ are the bias and input respectively. Figure 3 depicts an illustration of neural network model used for link classification in the flying ad-hoc network.

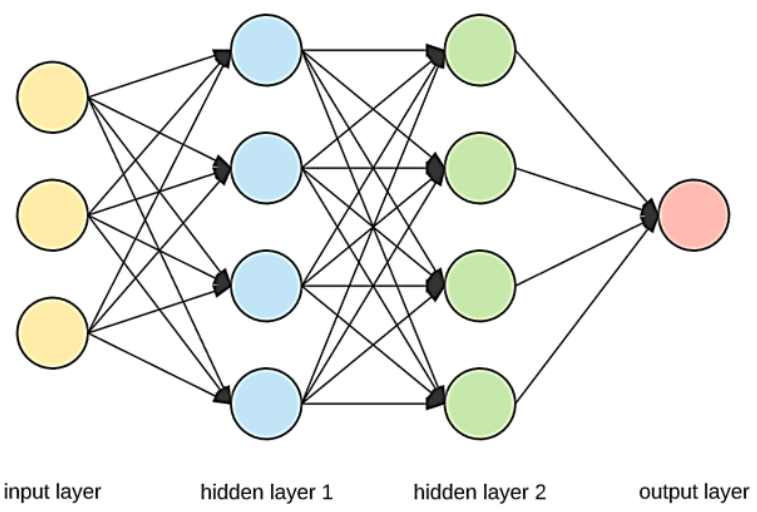

Fig. 3 ANN Architecture

This classification algorithm computes the present state and output state status and error is computed based on the threshold value. In order to minimize the error in the neural network weight factors are introduced. The learning process of the neural network is given as

$$
h(t+1)=h(t)+\alpha \frac{e_{p}(t) x(t)}{|x(t)|^{2}}
$$

The mathematical formulation of bee colony optimization is depicted in figure 4 which describes the dance area of the bee and the honey comb details. The nectar details are formulated for the source and destination. 
Vol.02/ No. 03

Pages: $155-164$

https://www.irojournals.com/tcsst/

DOI: https://doi.org/10.36548/jtcsst.2020.3.005

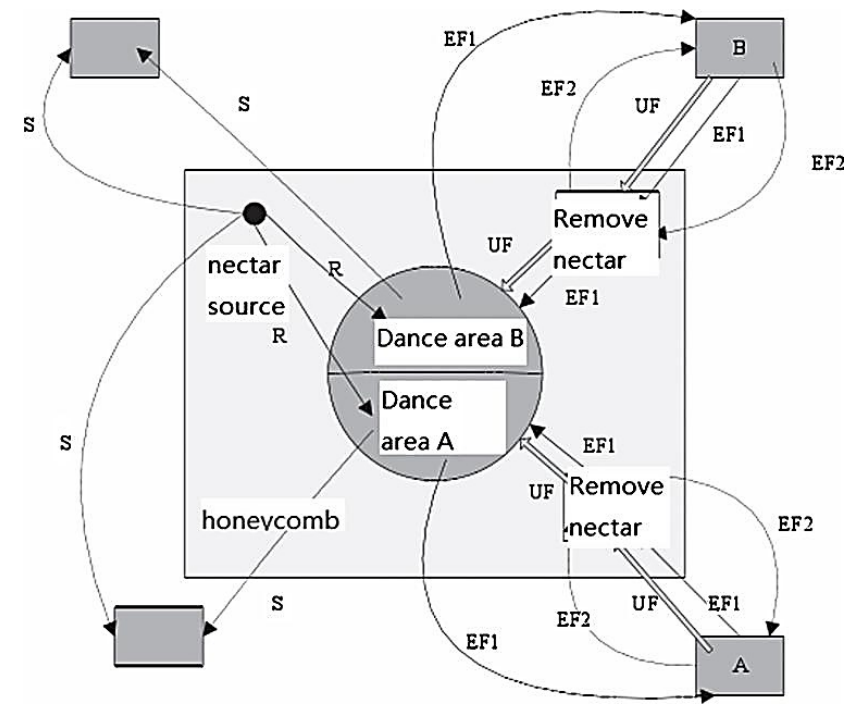

Fig. 4 Bee colony optimization model

In order to optimize the classification results of neural network model further a nature inspired artificial bee colony is used. Based on the food collecting strategy and finding optimal food source these bee colony optimization is formulated and it is widely used in many applications. Food searching and identifying optimal food location are the primary objective in bee colony optimization. Considering these evolutionary process, the link establishment and link management is developed in the proposed model using bee colony optimization. Based on the probability of bee movement the solutions are generated and it is given as

$$
\operatorname{Prob}_{v}^{n+1}=\exp ^{-\frac{\text { object }_{\text {max }}-\text { object }_{\text {norm }}}{v}}
$$

where object $_{\max }$ is the obtained solution which is maximized and the object $t_{\text {norm }}$ is the objective function which is normalized for the complete solution, $v$ is the pass value which is generally $1,2,3 \ldots$ Block diagram of the proposed model is depicted in figure 5 and the process of link establishment and link maintenance is described.

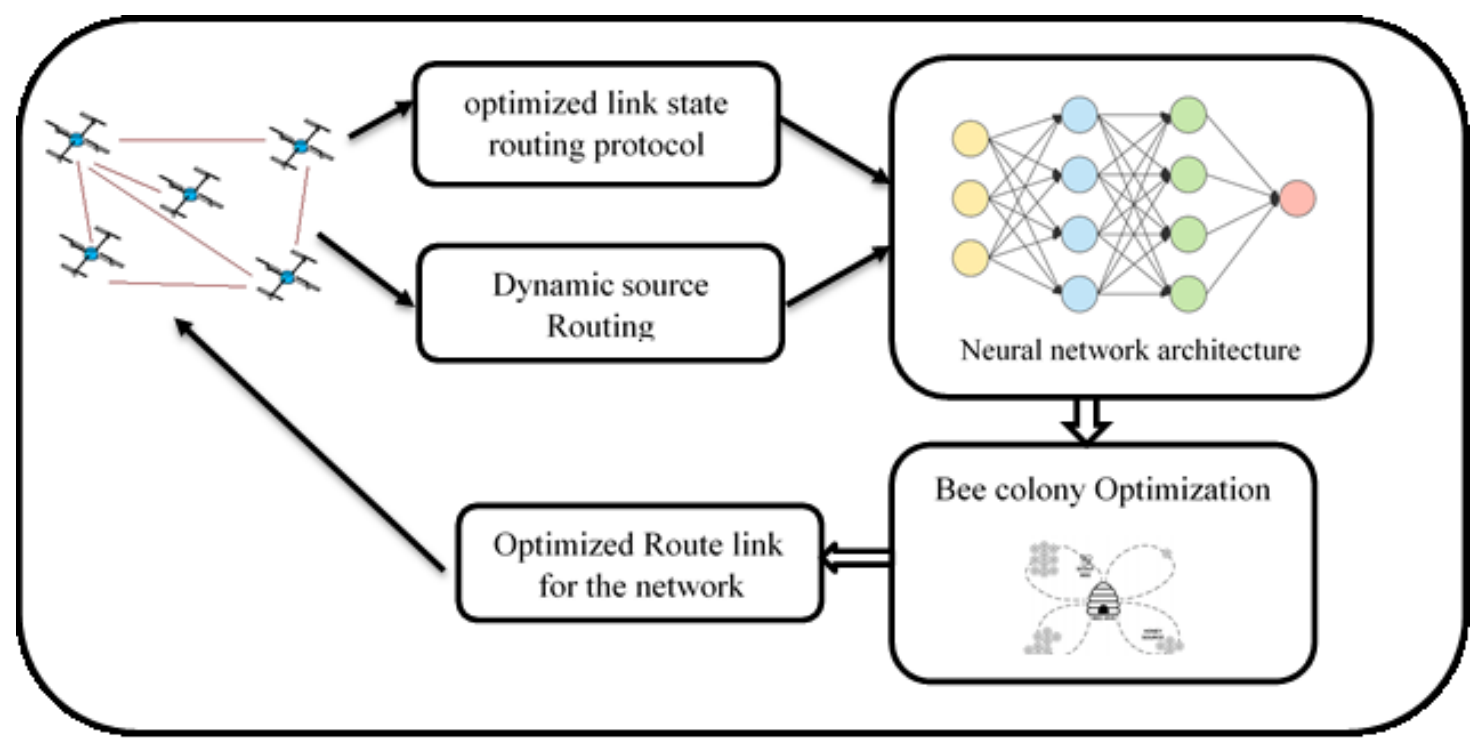

Fig.5 Proposed Hybrid Optimization model 
Journal of trends in Computer Science and Smart technology (TCSST) (2020)

Vol.02/ No. 03

Pages: $155-164$

https://www.irojournals.com/tcsst/

DOI: https://doi.org/10.36548/jtcsst.2020.3.005

The process starts from link request from source node and once the request is received the destination location is verified. Based on the location the routing strategy is selected as proactive or reactive and then it is classified using neural network model. The classified results are further optimized through bee colony optimization and provides link status to the network for effective link establishment and link management. The results of neural network model have various possibilities for link establishment and nature inspired optimization selects the best route from source to destination similar to its food searching behaviour and shares the status to the network. Once the optimized path is identified, connection is established between the source node to destination node and the data transfer process is initiated between the nodes.

\section{Result and Discussions}

The proposed hybrid model is experimented in Network Simulator 2 version 2.35 installed on Ubuntu. In order to highlight the superior performance of proposed model, conventional AODV and DSDV models are compared. The network parameters used for simulation is given in table 2.

Table 2. Simulation Network Parameters

\begin{tabular}{|c|c|c|}
\hline S.No & Parameters & Values \\
\hline 1 & Simulator & NS-2 (v2.35) \\
\hline 2 & Protocol & OLSR, DSR \\
\hline 3 & Simulation Area & $1000 x 1000$ \\
\hline 4 & Number of Nodes & 15 \\
\hline 5 & Data size & 2512 bytes $/$ packet \\
\hline 6 & Max No. of CBR Connections & $5,10,15,20,25,30,35(\mathrm{~m} / \mathrm{sec})$ \\
\hline 7 & Node Speed & \\
\hline
\end{tabular}

The performance of proposed model is measured based on the packet delivery ratio, end to end delay, throughput and communication overhead. Packet delivery ratio is measured based on the percentage of successful delivered packets with respect to the received packets. The performance comparison of proposed design in terms of packet delivery ratio is depicted in figure 6 and it is observed that the proposed model attains better performance with respect to varying node speed compared to other models.

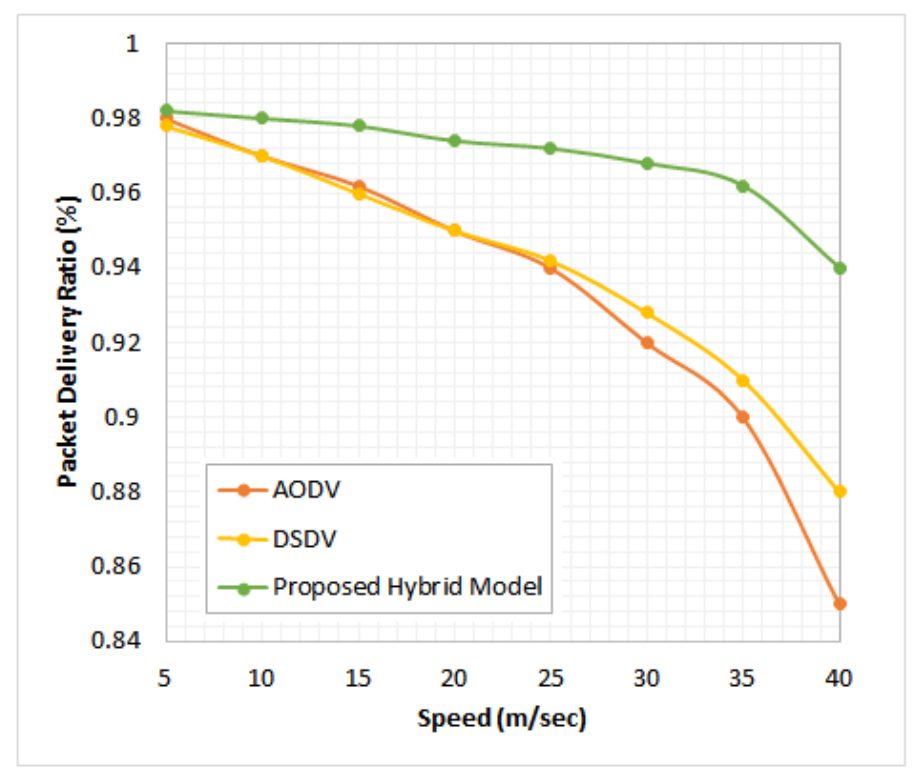

Fig. 6 Packet Delivery Ratio 
Journal of trends in Computer Science and Smart technology (TCSST) (2020)

Vol.02/ No. 03

Pages: $155-164$

https://www.irojournals.com/tcsst/

DOI: https://doi.org/10.36548/jtcsst.2020.3.005

The end to end delay analysis is used to measure the time taken for a packet to reach the destination from source which includes the node discovery time and time taken to complete the delivery process. Figure 7 depicts the comparison of end to end delay analysis of proposed model and other models. It is observed that proposed model has minimum delay time compared to conventional models.

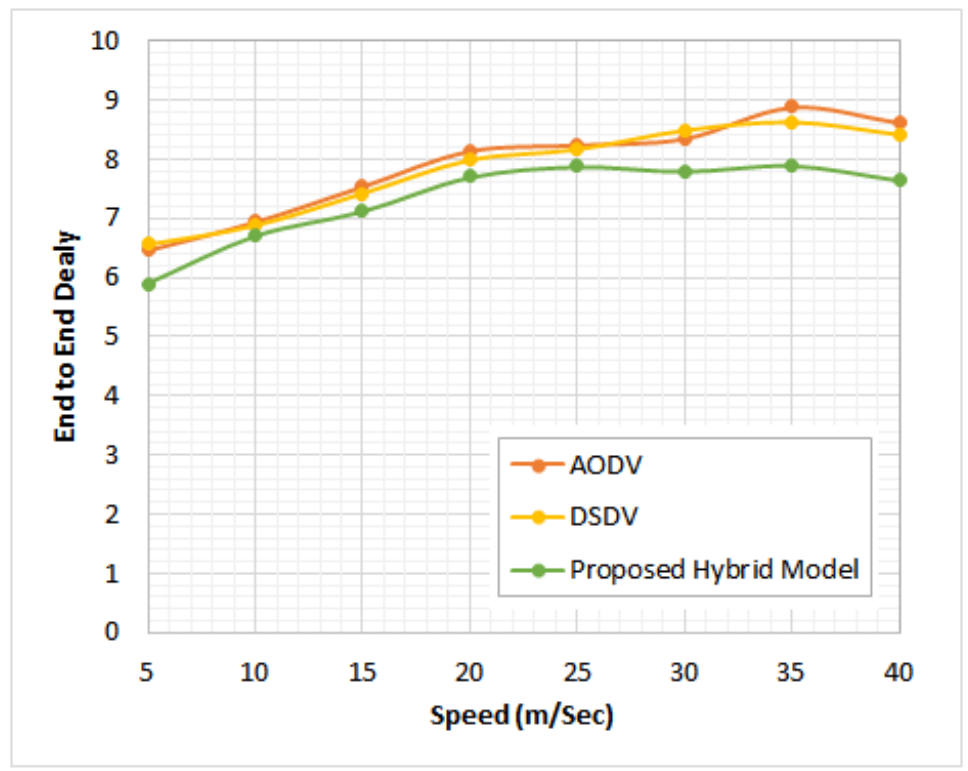

Fig. 7 End to end Delay analysis

Communication overhead of the proposed model is compared with conventional models and it is depicted in figure 8. It is observed that the proposed model has minimum overhead ratio compared to other models due to its dynamic routing strategy.

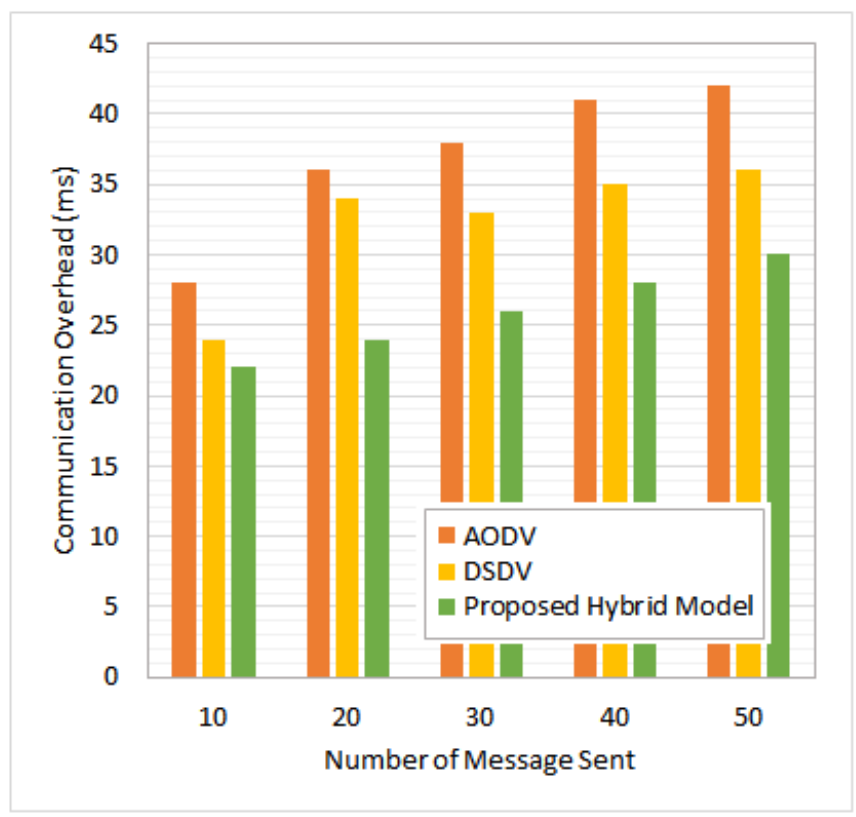

Fig. 8 Communication Overhead analysis

The successful delivery of the packets from source to destination with in the stipulated time is considered as throughput of the network. Figure 9 gives such throughput comparison of the proposed model with other models based on the node speed. It is observed that proposed model attains an average throughput for all the node speeds 
Journal of trends in Computer Science and Smart technology (TCSST) (2020)

Vol.02/ No. 03

Pages: $155-164$

https://www.irojournals.com/tcsst/

DOI: https://doi.org/10.36548/jtcsst.2020.3.005

while the other models have variations when the node speed increases. Compared to the performance of AODV the proposed model attains an average of 3\% increased throughput and $2.5 \%$ over DSDV routing algorithm.

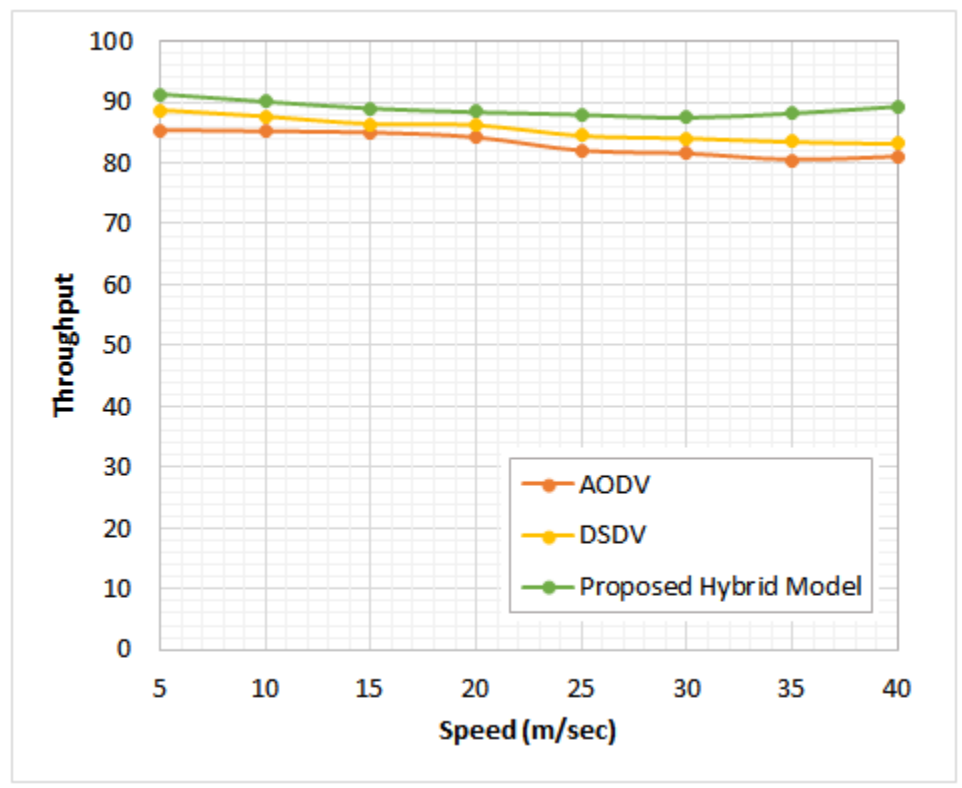

Fig. 9 Throughput Analysis

From the results it is observed that the proposed hybrid model attains better performance in terms of packet delivery ratio, end to end delay, throughput and communication overhead compared to conventional models like AODV and DSDV.

\section{Conclusion}

An efficient hybrid optimized routing scheme is introduced in this research work for flying ad-hoc networks. Most of the existing research models are focused over mobility in flying ad-hoc networks and not considered other parameters like throughput, communication overhead and packet delivery ratio. In order to improve the performance of network under dynamic and static situation this hybrid model is designed using optimized link state routing and dynamic source routing. Artificial neural network is used in the proposed design for link state analysis and nature inspired bee colony optimization is used to obtain the shortest path from source to destination from classification results. The experimentation results demonstrate that proposed model attains better performance in all parameters compared to conventional models. Compared to other routing models the cost function is slightly high and that is considered as a limitation of the proposed design. The future work could be progressed for improved performance with minimized cost using hybrid optimization models.

\section{References}

1. İlker Bekmezci, Ozgur Koray Sahingoz, Şamil Temel (2013). Flying Ad-Hoc Networks (FANETs): A survey. Ad Hoc Networks. 11(3):1254-1270

2. Chaker Abdelaziz Kerrache, Safdar Hussain Bouk, Rasheed Hussain, Syed Hassan Ahmed (2019). Introduction to the Special Section on Recent Advancements in Flying ad hoc Networks. Computers \& Electrical Engineering. 77,409-411.

3. Vishal Sharma, Ravinder Kumar, Neeraj Kumar (2018). Distributed priority tree-based routing protocol for FANETs. Computer Communications. 122, 129-151.

4. Wajiya Zafar, Bilal Muhammad Khan (2017). A reliable, delay bounded and less complex communication protocol for multicluster FANETs. Digital Communications and Networks. 3(1):30-38. 
Journal of trends in Computer Science and Smart technology (TCSST) (2020)

Vol.02/ No. 03

Pages: $155-164$

https://www.irojournals.com/tcsst/

DOI: https://doi.org/10.36548/jtcsst.2020.3.005

5. Ashish Khanna, Joel J. P. C. Rodrigues, Naman Gupta, Abhishek Swaroop, Victor Hugo C. de Albuquerque (2019). A mutual exclusion algorithm for flying Ad Hoc networks. Computers \& Electrical Engineering. 76, 82-93.

6. Rayner M. Pires, Alex Sandro Roschildt Pinto, Kalinka Regina Lucas Jaquie Castelo Branco (2019). he Broadcast Storm Problem in FANETs and the Dynamic Neighborhood-Based Algorithm as a Countermeasure. IEEE Access, 7,59737-59757.

7. Min Zhang, Chao Dong, Yang Huang (2019). FS-MAC: An Adaptive MAC Protocol with Fault-Tolerant Synchronous Switching for FANETs. IEEE Access, 7, 80602-80613.

8. Inam Ullah Khan, Ijaz Mansoor Qureshi, Muhammad Adnan Aziz, Tanweer Ahmad Cheema, Syed Bilal Hussain Shah (2020). Smart IoT Control-Based Nature Inspired Energy Efficient Routing Protocol for Flying Ad Hoc Network (FANET). IEEE Access, 8, 56371-56378.

9. Ali Khan, Farooq Aftab, Zhongshan Zhang (2019). BICSF: Bio-Inspired Clustering Scheme for FANETs," IEEE Access, 7, 31446-31456.

10. Imtiaz Mahmud, You-Ze Cho (2019). Adaptive Hello Interval in FANET Routing Protocols for Green UAVs. IEEE Access, 7, 63004-63015.

11. Armir Bujari, Claudio E. Palazzi, Daniele Ronzani (2018). A Comparison of Stateless Position-based Packet Routing Algorithms for FANETs," IEEE Transactions on Mobile Computing, 17(11):2468-2482.

12. Jiaxin Chen, Yuhua Xu, Qihui $\mathrm{Wu}$, Yuli Zhang, Xueqiang Chen, Nan Qi (2019). Interference-Aware Online Distributed Channel Selection for Multicluster FANET: A Potential Game Approach. IEEE Transactions on Vehicular Technology, 68(4):3792-3804.

13. Nishat I. Mowla, Nguyen H. Tran, Inshil Doh, Kijoon Chae (2020). AFRL: Adaptive federated reinforcement learning for intelligent jamming defense in FANET. Journal of Communications and Networks, 22(3):244-258.

14. Yixin He, Xiao Tang, Ruonan Zhang, Xiaojiang Du, Deyun Zhou, Mohsen Guizani (2019). A CourseAware Opportunistic Routing Protocol for FANETs. IEEE Access, 7, 144303-144312, 2019. 